EUROPEAN ORGANIZATION FOR NUCLEAR RESEARCH

European Laboratory for Particle Physics

Large Hadron Collider Project

LHC Project Report 105

\title{
THE SHORT STRAIGHT SECTIONS FOR THE LHC
}

\author{
T. Tortschanoff, V. Parma, P. Rohmig* \\ M. Peyrot, J.M. Rifflet, P.Védrine, CEA-Saclay, France and D. Vincent
}

\begin{abstract}
During more than five years a close collaboration between CERN and CEA-Saclay led to the development and construction of two prototype quadrupole magnets and the integration of one of them into the short straight section of the LHC half-cell test string at CERN. In the frame of the special host country contribution to the LHC project this collaboration has been extended to the CNRS laboratory in Orsay and covers besides the quadrupole magnets the complete cold mass assembly (CEA) and the integration into the short straight section cryostat (CNRS). The short straight sections include not only the main lattice quadrupoles with their protection diodes, they also house different corrector magnets and the beam position monitors. Further, they provide the cryogenic feed units for a half-cell with all the magnet interconnections and the jumper connection to the separate cryo-line. The paper will show the general lay-out of these complex units and elaborate the different aspects of their assembly.
\end{abstract}

PAC'97, Vancouver

Administrative Secretariat

LHC Division

CERN

CH - 1211 Geneva 23

Switzerland

Geneva, 30 May 1997 


\title{
THE SHORT STRAIGHT SECTIONS FOR THE LHC
}

\author{
T. Tortschanoff, V. Parma, P. Rohmig, CERN, Geneva, Switzerland, \\ M. Peyrot, J.M. Rifflet, P.Védrine, CEA-Saclay, France and D. Vincent CNRS, Orsay, France
}

\begin{abstract}
During more than five years a close collaboration between CERN and CEA-Saclay led to the development and construction of two prototype quadrupole magnets and the integration of one of them into the short straight section of the LHC half-cell test string at CERN. In the frame of the special host country contribution to the LHC project this collaboration has been extended to the CNRS laboratory in Orsay and covers besides the quadrupole magnets the complete cold mass assembly (CEA) and the integration into the short straight section cryostat (CNRS). The short straight sections include not only the main lattice quadrupoles with their protection diodes, they also house different corrector magnets and the beam position monitors. Further, they provide the cryogenic feed units for a half-cell with all the magnet interconnections and the jumper connection to the separate cryo-line. The paper will show the general lay-out of these complex units and elaborate the different aspects of their assembly.
\end{abstract}

\section{INTRODUCTION}

The major effort in the development of the LHC magnet system has been put into the design and construction of the dipole magnets. Since 1989, however, a collaboration with CEA-Saclay in France was started for the development of the lattice quadrupole magnets for the LHC. This resulted in the successful construction and testing of two prototype quadrupole magnets [1] of which one has been integrated into a short straight section unit forming the first cryo-magnet unit in CERN's test string operated since 1994 [2].

Following the approval of the LHC project a new collaboration for the design, prototyping and industrial series production was concluded between CERN and two French laboratories, CEA-Saclay and CNRS in Orsay. While the CEA is looking after the cold masses of the short straight sections CNRS has been entrusted with the work for the cryostating of these units.

Although much shorter than the $15 \mathrm{~m}$ long dipole units [3] the short straight sections are of much higher complexity. As their main magnetic component they contain the $3.25 \mathrm{~m}$ long twin aperture lattice quadrupole magnets. Further, on one end of the quadrupole the combined sextupole-dipole corrector magnets for each beam are placed, while on the other end a pair of octupole corrector magnets is mounted or, depending on their location in the arc, a pair of tuning quadrupoles. For the short straight sections which will be located in the dispersion suppresser regions and near the insertion points other types of correctors will be used like long tuning quadrupoles, skew quadrupoles or eventually skew octupole magnets.

\section{LAY-OUT OF THE SHORT STRAIGHT SECTIONS FOR LHC}

The cold mass of the earlier short straight section designed for the LHC test string consisted of two parts. One, the main twin aperture quadrupole magnet with its stiffening inertia tube, the other with all the correction elements, placed in a separate inertia tube: The two halves were aligned and joined together, both mechanically and electrically to form the cold mass of the prototype straight section.

The new LHC design features now among other modifications a symmetric arrangement of the quadrupoles with respect to the dipole magnets. This implies that the corrector magnets have to be on both sides of the quadrupole. Furthermore, the quadrupoles are powered independently from the dipoles thus reducing the integrated length of the tuning quadrupoles significantly. The inertia tube into which all magnets must be aligned, will no more be made out of two halves but in one single piece. It has to be rigid enough to guaranty the geometrical stiffness under the weight load and in all operating conditions.

The cryostat which has been simplified with respect to earlier versions [4] by the implementation of a separate cryogenic feed line, will be completed on one end by the cryogenic technical service module. The functions of this module are multiple. Each module will house the beam position monitors, the protection diodes of the quadrupole magnets and the cold mass instrumentation capillaries. Every second will contain in addition the cryogenic interconnection and the liquid helium separator. On the opposite end of the short straight section the interconnection is practically identical to those of the standard dipole magnets.

An important feature of the short straight section design is the sectorisation of the insulation vacuum [5]. This implies that the short straight section has a vacuum barrier which in the present design will be mounted every second cell, i.e., every fourth short straight section. This helps in the leak testing during installation and makes short intervention, like the exchange of a diode, possible. It permits in case of a major intervention, like the replacing of a magnet, to warm up one sector only. The liquid helium flow in between cold masses is also sectored 
by means of pressure plugs placed in the bus-bar connection tubes for every second cell.

\section{MAIN QUADRUPOLE MAGNETS}

The new quadrupole design follows the line of the earlier concept. The main parameters of this twin aperture magnet are summarised in table 1 .

Table 1: LHC Main Quadrupoles Parameters

\begin{tabular}{|c|c|c|c|}
\hline Nominal temperature & 1.9 & K & \\
\hline $\begin{array}{l}\text { Nominal gradient } \\
\qquad \mathrm{T} / \mathrm{m}\end{array}$ & \multicolumn{3}{|c|}{223} \\
\hline Margin on load line & \multicolumn{3}{|c|}{19.7} \\
\hline Nominal Current & \multicolumn{3}{|c|}{11870} \\
\hline Magnetic length & 3.10 & $\mathrm{~m}$ & \\
\hline Beam separation distance (cold) & 194.0 & $\mathrm{~mm}$ & \\
\hline $\begin{array}{l}\text { Inner coil aperture diameter (warm) } \\
\qquad \mathrm{mm}\end{array}$ & \multicolumn{3}{|c|}{56} \\
\hline $\begin{array}{l}\text { Outer coil diameter } \\
\qquad \mathrm{mm}\end{array}$ & \multicolumn{3}{|c|}{118.6} \\
\hline $\begin{array}{l}\text { Outer yoke diameter } \\
\qquad \mathrm{mm}\end{array}$ & \multicolumn{3}{|c|}{452} \\
\hline Collar material & \multicolumn{3}{|c|}{ Stainless steel } \\
\hline Yoke length including end plates & 3250 & $\mathrm{~mm}$ & \\
\hline
\end{tabular}

The super-conducting cable of the quadrupole magnets will be the same as the key-stoned cable used for the outer coil layer in the main dipole magnets. By this it is hoped to achieve savings not only because of standardisation but especially since the unit cable length for a quadrupole coil is considerably shorter than that for the dipoles.

Fig. 1 shows the cross-section of the quadrupolein its cryostat. Since the electromagnetic forces in a quadrupole are significantly lower than in a dipole no contribution of the yoke is needed to pre-stress the coils. This is ensured by the independent stainless steel collars which themselves are centred inside the single piece yoke laminations by longitudinal keys. The cut-off on top or bottom of every second yoke laminations allows to press and key them such that the coil collar units are well fixed and aligned inside the yoke apertures. The centring of the yoke inside the inertia tube is again provided by a series of keys which are blocked from the outside of the tube by a series of bolts. Welded covers over these series of bolts have to make sure that the inertia tube is helium leak tight to an operational maximum pressure of 20 bar and fulfils its function as helium vessel. The upper of the two $60 \mathrm{~mm}$ diameter holes in the yoke will house the heat exchanger tube which runs through all elements of one machine cell and where the cooling down to $1.9 \mathrm{~K}$ takes place [6]. The lower one will be used for the passage of cables, especially for instrumentation and eventually for corrector magnet wiring.

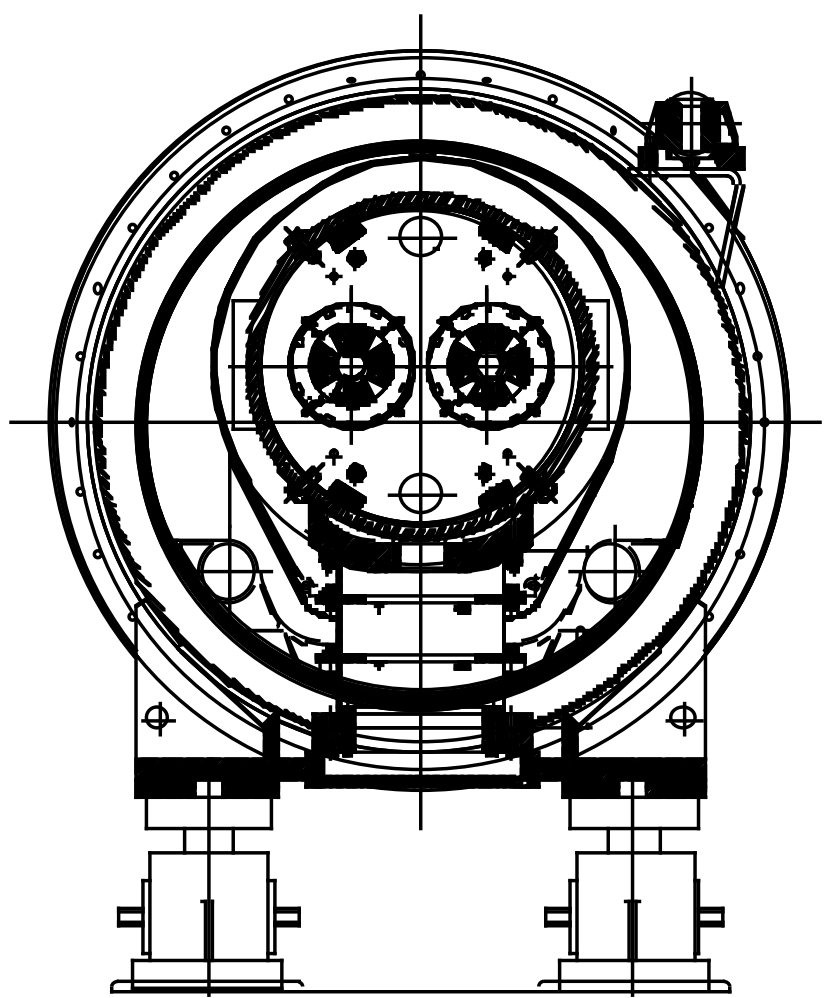

Figure 1 - Cross-section of the LHC lattice quadrupole in its short straight section cryostat

\section{CORRECTOR MAGNETS}

Each short straight section will have on the end, opposite to the connections, the sextupole- dipole corrector magnets. While the sextupole coils of this combined units will be powered in series to currents up to nominally $500 \mathrm{~A}$ the corrector dipole magnets, alternatively either horizontally or vertically deflecting ones, will be powered individually which means that they have to have their current passage between $1.9 \mathrm{~K}$ and room temperature in every short straight section. Their maximum operational current will be $32 \mathrm{~A}$. The yoke length of these units will be $1.26 \mathrm{~m}$, the sextupole strength is $1500 \mathrm{~T} / \mathrm{m}^{2}$ over a magnetic length of $1.10 \mathrm{~m}$ the corrector dipole field is $1.5 \mathrm{~T}$ over $1.03 \mathrm{~m}$. These coils will be assembled in a common yoke and fixed inside the inertia tube similar to the main quadrupoles.

On the connection side of the quadrupole, most short straight sections will have two octupole corrector magnets of $380 \mathrm{~mm}$ overall length, including a magnetic shield around them. Their strength will be $6.7 .10^{4} \mathrm{~T} / \mathrm{m}^{3}$ over a magnetic length of $320 \mathrm{~mm}$. Their outer diameter will not be more than $117 \mathrm{~mm}$ and thus they will be mounted separately and fixed by a pair of plates inside the inertia tube. The nominal current will be $600 \mathrm{~A}$.

The short straight sections near the ends of the arc will have tuning quadrupoles in place of the octupoles. With the same amperage and overall length they will have a larger outer diameter of $170 \mathrm{~mm}$. Their field gradient will be $120 \mathrm{~T} / \mathrm{m}$. In some of the short straight sections in 
the dispersion suppresser region their yoke length will be $1700 \mathrm{~mm}$. Because of their high number and their similitude to the corrector magnets in the dipoles cold masses a semi-automized coil fabrication is envisaged for all corrector magnet coils.

\section{CRYOSTAT}

The short straight section cryostat has been conceived together with the dipole cryostat. With the separated cryo line in the tunnel its cross-section had been simplified considerably. As a variation to the dipole cryostat the sectorisation must be mentioned. This item will have to take not only differences in pressure and assure leak tightness it must also allow for all the dimensional variations during cool-down and operation. Further, it must be mountable in the short straight section cryostats where needed and interface well with the cryostat, the cold mass, the tubes and the shielding inside the cryostat. Two techniques have been pursued, one with an all steel membrane and one with a composite membrane in collaboration with industry [7].

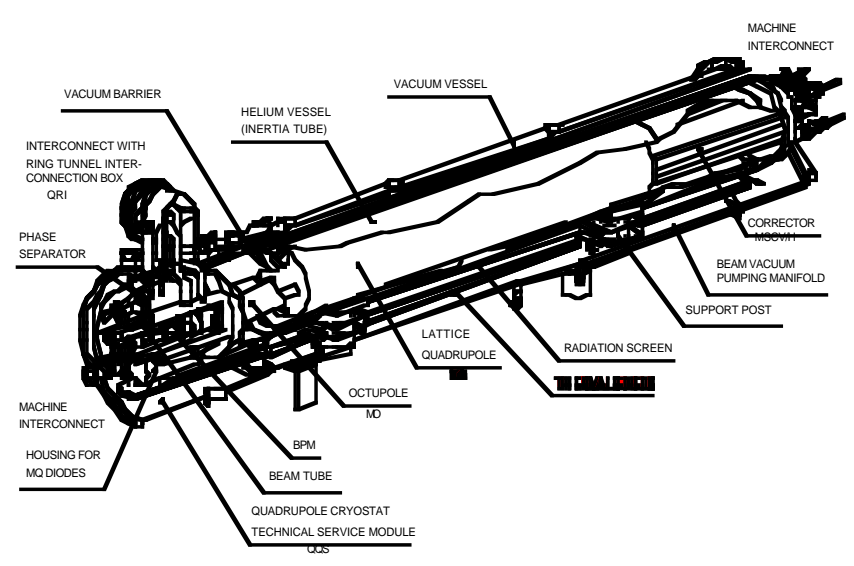

Figure 2 - Perspective view of the cold mass in cryostat together with cryogenic technical service module

Only two cryogenic pipes remain inside the cryostat which are the one for thermalising the thermal shield at $50 \mathrm{~K}$ and that for thermalising the upper part of the support posts to $5-10 \mathrm{~K}$. One of the two support posts, positioning the cold mass inside the cryostat, will be fixed, the other one will be allowed to slide to compensate thermal contraction. Fig. 2 shows a perspective view of the cold mass in the cryostat.

\section{CRYOGENIC TECHNICAL SERVICE MODULE}

The short straight section is completed by a cryogenic technical service module. This will contain the two beam position monitors attached to their beam screens and bolted to the endplate of the cold mass, the quench protection diodes in their container. Every second module will have its cryogenic jumper connection to the separate cryogenic supply line and a helium phase separator. which provides for all cryogenic but also electrical interconnections. This implies that there will be two major types of these modules.

The magnet to magnet linking must provide the continuity of the currents and helium flow or of the plugging of the latter one where required. In this area will also be placed the interconnections for the auxiliary busbars and their leads to the corrector magnets of the short straight section in question. Further, there must be passages of the instrumentation wiring from the coldmass interior to the capillaries which leads them to outside of the cryostat. All this makes this service module a very complex unit the study of which is not yet finalised.

\section{CONCLUSIONS}

The short straight sections of the LHC are vital units for the operation of the machine. Their complexity is given by the multitude of magnetic and other machine components. The presence of the cryogenic technical service module with its variants and different combinations of function complicates the still ongoing design even further. CERN is however confident to realise these units in the great number of their different versions with the active help of the collaborating laboratories, CEA-Saclay and CNRS in Orsay.

\section{REFERENCES}

[1] K. Henrichsen et al., Cryogenic and mechanical measurements of the first two LHC lattice quadrupole prototypes, Proc. EPAC 94, London, UK, 27 June to 1 June 1994.

[2] A. Bézaguet et al., The LHC test string: first operational experience, Proc. EPAC 96, Sitges, Barcelona, Spain, 10 to 14 June 1996.

[3] R. Perin, Super-conducting magnets for the Large Hadron Collider, Proc. EPS 10, Trends in Physics, Seville, Spain, 3 to 13 Sep. 1996.

[4] W. Cameron et al., Design and construction of a prototype super-fluid helium cryostat for the short straight section of the CERN Large Hadron Collider (LHC), Proc. Int. Cryogenic Engineering Conf. (CEC), Albuquerque, NM, USA, 12 to 16 July 1993.

[5] M. Bona et al., Cryogenic and vacuum sectorisation of the LHC arcs, CERN-LHC-Project Report 60.

[6] V. Benda et al., Conceptual design of the cryogenic system for the Large Hadron Collider (LHC), Proc. EPAC 96, Sitges, Spain, 10 to 14 June 1996.

[7] B. Jenny, P. Rohmig, J.-M. Uriarte, A Composite Vacuum Barrier for the LHC Short Straight Section, Proc. Cryogenic Engineering Conf. (CEC), Columbus-Ohio, USA, 17 to 21 July 1995. 
